\title{
Biallelic MUTYH mutations can mimic Lynch syndrome
}

\author{
Monika Morak ${ }^{1,2}$, Barbara Heidenreich ${ }^{1}$, Gisela Keller ${ }^{3}$, Heather Hampel ${ }^{4}$, Andreas Laner ${ }^{2}$, \\ Albert de la Chapelle ${ }^{4}$ and Elke Holinski-Feder ${ }^{\star, 1,2}$
}

The hallmarks of Lynch syndrome (LS) include a positive family history of colorectal cancer (CRC), germline mutations in the DNA mismatch repair (MMR) genes, tumours with high microsatellite instability (MSI-H) and loss of MMR protein expression. However, in $\sim 10-15 \%$ of clinically suspected LS cases, MMR mutation analyses cannot explain MSI-H and abnormal immunohistochemistry (IHC) results. The highly variable phenotype of $M U T Y H$-associated polyposis (MAP) can overlap with the LS phenotype, but is inherited recessively. We analysed the MUTYH gene in 85 'unresolved' patients with tumours showing IHC MMR-deficiency without detectable germline mutation. Biallelic p.(Tyr179Cys) MUTYH germline mutations were found in one patient (frequency $1.18 \%$ ) with CRC, urothelial carcinoma and a sebaceous gland carcinoma. LS was suspected due to a positive family history of CRC and because of MSI-H and MSH2-MSH6 deficiency on IHC in the sebaceous gland carcinoma. Sequencing of this tumour revealed two somatic MSH2 mutations, thus explaining MSI-H and IHC results, and mimicking LS-like histopathology. This is the first report of two somatic MSH2 mutations leading to an MSI-H tumour lacking MSH2-MSH6 protein expression in a patient with MAP. In addition to typical transversion mutations in KRAS and APC, MAP can also induce tumourigenesis via the MSI-pathway.

European Journal of Human Genetics (2014) 22, 1334-1337; doi:10.1038/ejhg.2014.15; published online 12 February 2014

Keywords: MUTYH; base excision repair; somatic mutations; Lynch syndrome; immunohistochemistry; DNA mismatch repair

\section{INTRODUCTION}

Lynch syndrome (LS) is the most frequent autosomal dominant predisposition for early-onset colorectal cancer (CRC) (MIM\# 114500) and associated tumours, ${ }^{1}$ and is diagnosed by the detection of a germline mutation in one of the mismatch repair (MMR) genes MLH1, MSH2, MSH6 or PMS2. In tumours, the typical clues for LS include high microsatellite instability (MSI-H) and the loss of MMR protein expression in immunohistochemistry (IHC). However, in $10-15 \%$ of our cases clinically suspected of LS, the MSI-H and abnormal IHC results in the tumours cannot be explained by MMR germline mutation analyses. ${ }^{2,3}$

Germline mutations in the human Mut Y homolog (MUTYH; MIM\# 604933) also lead to a predisposition to CRC and adenomas but with an autosomal recessive inheritance. The clinical picture of MUTYH-associated polyposis (MAP, MIM' 608456) is extremely variable and ranges from severe polyposis coli to attenuated forms with late age of onset or few adenomas or CRC, which creates phenotypic overlap with LS. ${ }^{4-9}$

So far, three MAP patients have been described in the literature with an IHC loss of MLH1 protein staining and an MSI-H tumour: one male with a biallelic MUTYH germline mutation c.1227_28dupGG; p.(Glu410Glyfs $\left.{ }^{\star} 43\right)$ affecting the base excision repair (BER), and one somatic mutation in the MLH1 gene detected in his left-sided CRC at age of 49 years, ${ }^{10}$ the second with two heterozygous MUTYH mutations c.536A>G; p.(Tyr179Cys) and c.1187G >A; p.(Gly396Asp) and biallelic methylation of the MLH1 promoter in her caecal carcinoma at the age of 50 years ${ }^{11}$ and the third with homozygous MUTYH mutation c.536A > G; p.(Tyr179Cys) and no analyses for methylation or somatic mutation in $\mathrm{MLH1}$ performed in the right-sided colon carcinoma diagnosed at age 50 years. ${ }^{12}$ In some publications CRC patients with MSI-H tumours were excluded for MUTYH analyses or MSI was not analysed. ${ }^{13,14}$ Three further MAP patients were identified with MSI-H tumours but no information on IHC for MMR proteins was given, ${ }^{15,16}$ whereas other studies detected no biallelic MUTYH mutation carrier in patients with MSI-H tumours. ${ }^{17-20}$ To investigate the role of MAP as a possible cause of somatic mutations in MMR genes we analysed MUTYH in blood DNA of 85 'unresolved' patients suspected of having LS due to IHC loss of MMR protein expression.

\section{MATERIALS AND METHODS}

The study cohort includes 85 patients suspected of having LS based on the MSI-H and pathological IHC results in their tumours: 23 had IHC loss of MLH1 and PMS2, 35 had loss of MSH2 and MSH6, 19 showed loss of MSH6 only, and 8 lacked expression of PMS2 only. All patients met at least one of the Bethesda criteria ${ }^{21}$ and were tested negative for germline mutations and deletions or duplications in the MLH1, MSH2, MSH6, EPCAM and PMS2 genes before this study. MLH1-deficient cases were included if $\mathrm{MLH1}$ promoter methylation was absent in tumour DNA. Sixty nine patients were from Germany and sixteen from the USA. All patients provided their informed consent for cancer genetics research.

${ }^{1}$ Medizinische Klinik und Poliklinik IV, Campus Innenstadt, Klinikum der Universität München, Munich, Germany; ${ }^{2}$ MGZ - Medizinisch Genetisches Zentrum, Munich, Germany; ${ }^{3}$ Institute of Pathology, Technical University of Munich, Munich, Germany; ${ }^{4}$ Human Cancer Genetics Program, Comprehensive Cancer Center, The Ohio State University, Columbus, $\mathrm{OH}$, USA

*Correspondence: Professor E Holinski-Feder, MGZ - Center of Medical Genetics, Bayerstr, Germany and Medizinische Klinik und Poliklinik IV, Campus Innenstadt, Klinikum der Universität München, 3-5, 80335 Munich, Ziemssenstr. 1, 80336 Munich, Germany. Tel: +49 8930908860 ; Fax: +49 89 3090 88666 ; E-mail: elkeholinski-feder@t-online.de Received 26 September 2013; revised 20 December 2013; accepted 16 January 2014; published online 12 February 2014 
Control cohort: 403 CRC patients with either confirmed LS by pathogenic MMR germline mutation $(N=326)$ or sporadic tumourigenesis by BRAF mutation and $M L H 1$ promoter methylation in tumour DNA $(N=77)$.

DNA extraction from EDTA blood, standard PCR and direct sequencing with ABI PRISM 3100 Avant (Big Dye v1.1) was performed according to standard protocols. For computer analysis of the sequences the software Mutation surveyor 3.1 (SoftGenetics, State College, PA, USA) was used.

Mutation pre-screening of MUTYH was performed by direct sequencing of exon 6-7 and 12-13 including the hotspot mutations p.(Tyr179Cys) and p.(Gly396Asp) after PCR amplification using Ampli-Taq Gold (Life Technologies, Carlsbad, CA, USA) and digestion with Exo-SAP kit (Affymetrix, Santa Clara, CA, USA). At least one of these two hotspot mutations is found in $82-98 \%$ of all MAP cases in Caucasian, Canadian, American and Australian populations ${ }^{12,22}$ and in our own unpublished cohort. In a further step, sequencing has been completed for all 16 coding exons and flanking regions of MUTYH (GenBank: NM_001128425.1; NG_008189.1) for 73 patients, 12 patients could not be analysed completely due to lack of DNA. In addition, deletion/duplication screening of all MUTYH exons was carried out by multiplex ligation-dependent probe amplification (MLPA) with MLPA kit P378-A1 Lot0910 (MRC Holland, Amsterdam, The Netherlands) and analysed with GeneScan 3.7 software (Life Technologies).

Owing to the accepted nomenclature, which uses the longest MUTYHtranscript (NM_001128425.1) as a reference, nucleotide and amino acid numbering after nucleotide position 157 (amino acid 53) may differ from other publications by up to 42 nucleotides ( 14 amino acids). The frequent mutations p.(Tyr179Cys) in exon 7 and p.(Gly396Asp) in exon 13, previously reported as p.(Tyr165Cys) and p.(Gly382Asp) correspond to p.(Tyr176Cys) and p.(Gly393Asp) using other transcripts of MUTYH.

Germline MUTYH mutations were submitted to the LOVD database (http:// chromium.liacs.nl/LOVD2/colon_cancer/home.php?select_db=MUTYH).

Tumour DNAs from formalin-fixed paraffin-embedded tissues available from the biallelic mutation carrier were sequenced for somatic MAP-specific G:C > T:A transversions in MSH2 (NM_000251.2; NG_007110.2), KRAS exon 2 and 3 (NM_004985.3; NG_007524.1), and BRAF exon 15 (NM_004333.3).

\section{RESULTS}

Biallelic MUTYH germline mutations lead to biallelic somatic transversions in the MMR gene $\mathrm{MSH} 2$

In one patient displaying loss of MSH2 and MSH6 on IHC of his sebaceous gland carcinoma, we detected homozygosity for the pathogenic MUTYH germline mutation c.536A $>$ G; p.(Tyr179Cys) in exon 7. A deletion of the second allele was excluded by MLPA. The patient was diagnosed with two synchronous rectal adenocarcinomas T2 N0 G2 and several tubulo-villous adenomas at age 55, an urothelial bladder carcinoma at age 65 , a sebaceous gland carcinoma and a sebaceous gland hypertrophy at age 66 years. The rectal cancer and the urothelial bladder cancer were microsatellite stable and had normal MMR protein staining by IHC.

LS was suspected originally due to the patient's personal history in addition to his family history of a brother with caecal CRC T3 N2 G3 at age 56 years and two affected cousins (CRC at age 50; breast cancer at age 41 years) (Figure 1). Biallelic copies of the MUTYH mutation c.536A > G; p.(Tyr179Cys) were also found in DNA extracted from the paraffin-embedded normal tissue of the deceased brother. DNA of further family members was not available for genetic testing to assess the MUTYH mutation status.

Chronic lymphadenitis as a typical histological feature of LS was described in the CRC of the index patient and his brother. Information on Crohn's-like lymphocytic response and tumour-infiltrating lymphocytes reported in BER- and MMR-deficient tumours ${ }^{16,23}$ was not given.

\section{Tumour analyses}

Tumour sequencing of the index patient's sebaceous gland carcinoma identified two somatic pathogenic transversion mutations in the MSH2 gene (c.2101G > T; p. $($ Glu701*) in exon 13 and c.2554G > T; p.(Glu852*) in exon 15, Supplementary Figure 1A) explaining the development of MSI-H in tumourigenes is and loss of the MSH2 and MSH6 proteins in this tumour. The phase of the MSH2 mutations in exon 13 and 15 in tumour DNA could not be determined, as we did not find heterozygous SNPs in the neighbouring genomic regions in blood DNA of the patient and the amplification of $4.6 \mathrm{~kb}$ from exon 13 to 15 in tumour DNA for subcloning was not successful due to the poor DNA quality. The other two tumours, a rectal carcinoma and an urothelial carcinoma that were microsatellite stable, both had a heterozygous somatic KRAS hotspot mutation c.34G $>$ T; p.(Gly12Cys) transversion in exon 2 (Supplementary Figure 1B).

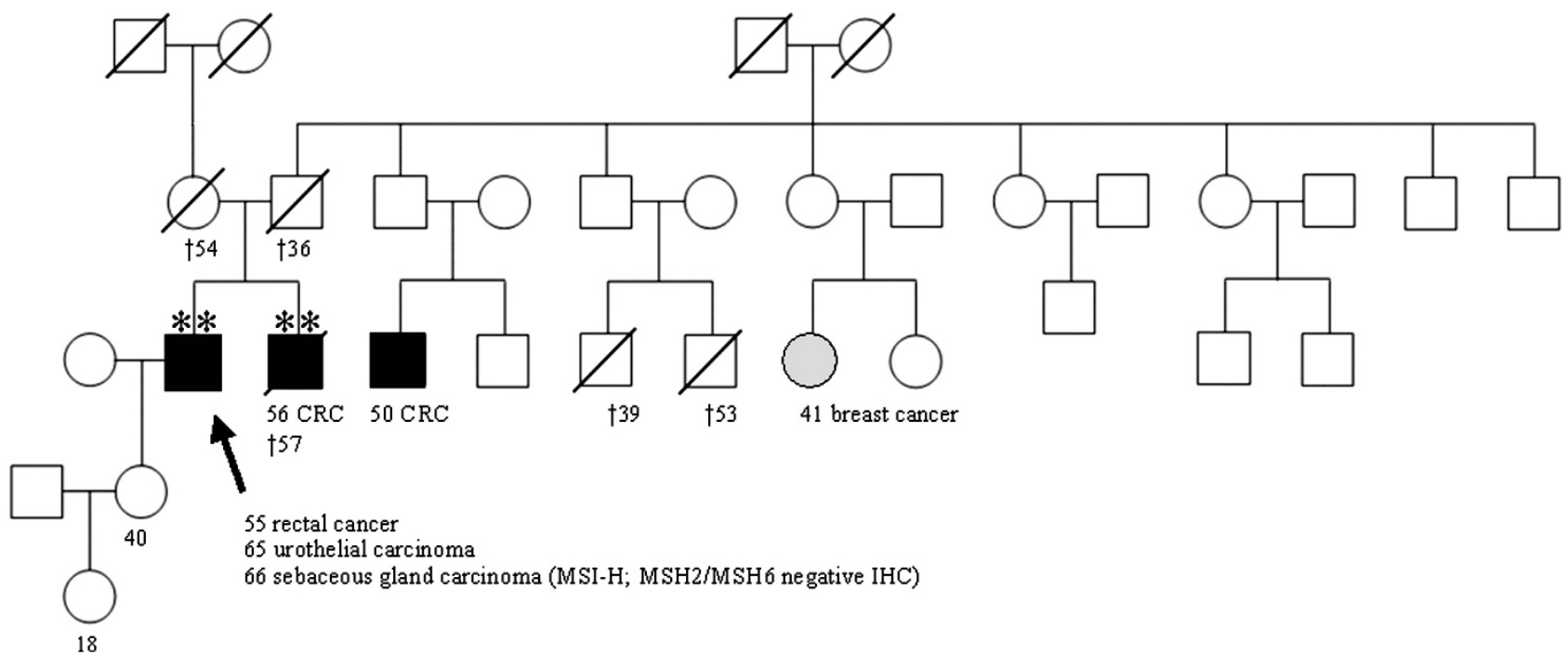

Figure 1 Pedigree of the family with the affected individual III:2 and his affected brother III:3 both bearing a homozygous MUTYH p.(Tyr179Cys) mutation ${ }^{(* *)}$. The sebaceous gland carcinoma revealed an MSI-H phenotype with loss of MSH2/MSH6 expression. Germline mutation analysis of MMR genes was negative. No genomic DNA was available from other affected family members with CRC as well as no detailed information concerning the parents. 


\section{Monoallelic MUTYH germline mutations in LS suspected} individuals and controls

None of the remaining patients analysed were found to have MUTYH mutations. The control cohort of 403 CRC patients revealed five patients with monoallelic heterozygous hotspot mutations (two in exon 7 and three in exon 13). No second MUTYH mutation was found in these patients.

\section{DISCUSSION}

Biallelic MUTYH mutations impair the BER process and can result in somatic mutational inactivation of both MMR-alleles mimicking LS by displaying a MMR-deficient tumour. To our knowledge, in the literature six MAP patients with MSI-H tumours were described, ${ }^{15,16}$ three of them with an IHC MLH1-deficiency in their MSI-H CRC. ${ }^{10-12}$ The loss of MLH1 staining in those three MAP tumours with MSI-H was caused by a somatic MAP-specific mutation in MLH1 together with a second undetectable defect, ${ }^{10}$ whereas biallelic methylation of the MLH1 promoter was causing the MLH1-deficiency in the other MAP patient. ${ }^{11}$

Here we present a MAP-patient, in which BER deficiency was associated with the development of two somatic MAP-specific G $>$ T transversion mutations in the $\mathrm{MSH} 2$ gene in the tumour resulting in MMR deficiency, MSI-H and a loss of MSH2 and MSH6 expression in the patient's sebaceous gland carcinoma. This patient's CRC and urinary bladder carcinoma revealed the MAP-specific KRAS G>T transversion mutations. ${ }^{14}$ The somatic $\mathrm{G}>\mathrm{T}$ transversions in $\mathrm{MSH} 2$ or KRAS, respectively, in three different tumours of the patient are very likely to be due to the germline BER defect. ${ }^{7,8}$

Interestingly, three further cancer diagnoses were reported in this family including CRC and breast cancer, which are compatible with a recessive mode of inheritance and the clinical phenotype of MAP. ${ }^{23-25}$ Biallelic MUTYH mutations were detected in the brother and might also have induced the tumourigenesis in the cousins (not investigated). Heterozygous MUTYH mutations were not identified in the remaining 84 index patients, for which somatic mutations or further germline pathomechanisms, for example, large genomic rearrangements such as inversions in the MMR genes have to be considered. ${ }^{3,26-28}$ In the control cohort the MUTYH mutation frequency of $1.24 \%$ was comparable to the frequencies of $0 \%-4.8 \%$ reported in the general population. ${ }^{12,22,29}$

As MAP can manifest in an extremely variable phenotype, MUTYH mutation analysis should also be considered in patients with a low number of adenomas, early-onset or synchronous or metachronous CRC and in patients with MSS tumours. ${ }^{30,31}$ With an incidence of only $1.18 \%$ in MMR-deficient CRC patients with typical histological features of LS and several adenomas MAP can mimic LS. These patients need different surveillance and due to the different pattern of inheritance a different risk annotation for family members. ${ }^{32} \mathrm{We}$ suggest to perform MUTYH genetic testing in these unresolved MSI-H cases with additional adenomas either in the two fragments harbouring the common mutations or in the course of nextgeneration sequencing cancer kits including the MMR genes, APC and MUTYH besides others. ${ }^{18}$

We reported for the first time a MAP patient initially suspected of LS, in which two MAP-specific somatic MSH2 mutations explained the IHC loss of MSH2/MSH6 in his MSI-H tumour. MAP might be a differential diagnosis for CRC patients with MSI-H tumours and several adenomas.

\section{CONFLICT OF INTEREST}

The authors declare no conflict of interest.

\section{ACKNOWLEDGEMENTS}

We would like to thank the German Cancer Aid (Deutsche Krebshilfe grant 110780) and the Wilhelm Sander-Stiftung grant 2012.081.1 as well as grants P30 16058 and P01 CA124570 from the National Cancer Institute, USA, for support of this work. We also thank all patients for their participation in this study, as well as their respective doctors for contributing clinical information.

1 Lynch HT, de la Chapelle A: Genetic susceptibility to non-polyposis colorectal cancer J Med Genet 1999; 36: 801-818.

2 Koehler U, Grabowski M, Bacher U, Holinski-Feder E: A new interphase fluorescence in situ hybridization approach for genomic rearrangements involving MLH1 and MSH6 in hereditary nonpolyposis colorectal cancer-suspected mutation-negative patients. Cancer Genet Cytogenet 2007; 175: 81-84.

3 Morak M, Koehler U, Schackert HK et al: Biallelic MLH1 SNP cDNA expression or constitutional promoter methylation can hide genomic rearrangements causing Lynch syndrome. J Med Genet 2011; 48: 513-519.

4 Aceto G, Cristina Curia M, Veschi S et al: Mutations of APC and MYH in unrelated Italian patients with adenomatous polyposis coli. Hum Mutat 2005; 26: 394.

5 Aretz S, Uhlhaas S, Goergens H et al: MUTYH-associated polyposis: 70 of 71 patients with biallelic mutations present with an attenuated or atypical phenotype. Int J Cancer 2006; 119: 807-814.

6 Sieber OM, Lipton L, Crabtree $\mathrm{M}$ et al: Multiple colorectal adenomas, classic adenomatous polyposis, and germ-line mutations in MYH. N Engl J Med 2003; 348: 791-799.

7 Al-Tassan N, Chmiel NH, Maynard J et al: Inherited variants of MYH associated with somatic G:C-> T:A mutations in colorectal tumors. Nat Genet 2002; 30: 227-232.

8 Jones S, Emmerson P, Maynard J et al: Biallelic germline mutations in MYH predispose to multiple colorectal adenoma and somatic G:C-> T:A mutations. Hum Mol Genet 2002; 11: 2961-2967.

9 Morak M, Laner A, Bacher U, Keiling C, Holinski-Feder E: MUTYH-associated polyposis - variability of the clinical phenotype in patients with biallelic and monoallelic MUTYH mutations and report on novel mutations. Clin Genet 2010; 78: 353-363.

10 Lefevre $\mathrm{JH}$, Colas $\mathrm{C}$, Coulet $\mathrm{F}$ et al: MYH biallelic mutation can inactivate the two genetic pathways of colorectal cancer by APC or MLH1 transversions. Fam Cancer 2010; 9: 589-594.

11 Colebatch A, Hitchins M, Williams R, Meagher A, Hawkins NJ, Ward RL: The role of MYH and microsatellite instability in the development of sporadic colorectal cancer. Br J Cancer 2006; 95: 1239-1243.

12 Cleary SP, Cotterchio M, Jenkins MA et al: Germline MutY human homologue mutations and colorectal cancer: a multisite case-control study. Gastroenterology 2009; 136: 1251-1260.

13 Knopperts AP, Nielsen M, Niessen RC et al: Contribution of bi-allelic germline MUTYH mutations to early-onset and familial colorectal cancer and to low number of adenomatous polyps: case-series and literature review. Fam Cancer 2013; 12 43-50.

14 van Puijenbroek M, Nielsen M, Tops CM et al: Identification of patients with (atypical) MUTYH-associated polyposis by KRAS2 c.34G $>$ T prescreening followed by MUTYH hotspot analysis in formalin-fixed paraffin-embedded tissue. Clin Cancer Res 2008; 14: 139-142.

15 Kambara T, Whitehall VL, Spring KJ et al: Role of inherited defects of MYH in the development of sporadic colorectal cancer. Genes Chromosomes Cancer 2004; 40: $1-9$.

16 O'Shea AM, Cleary SP, Croitoru MA et al: Pathological features of colorectal carcinomas in MYH-associated polyposis. Histopathology 2008; 53: 184-194.

17 Giraldez MD, Balaguer F, Bujanda L et al: MSH6 and MUTYH deficiency is a frequent event in early-onset colorectal cancer. Clin Cancer Res 2010; 16: 5402-5413.

18 Tanskanen T, Gylfe AE, Katainen R et al: Exome sequencing in diagnostic evaluation of colorectal cancer predisposition in young patients. Scand J Gastroenterol 2013; 48: 672-678.

19 Wang L, Baudhuin LM, Boardman LA et al: MYH mutations in patients with attenuated and classic polyposis and with young-onset colorectal cancer without polyps. Gastroenterology 2004; 127: 9-16.

20 Urso E, Agostini M, Pucciarelli S et al: Clinical and molecular detection of inherited colorectal cancers in northeast Italy: a first prospective study of incidence of Lynch syndrome and MUTYH-related colorectal cancer in Italy. Tumour Biol 2012; 33: 857-864.

21 Umar A, Boland CR, Terdiman JP et al: Revised Bethesda Guidelines for hereditary nonpolyposis colorectal cancer (Lynch syndrome) and microsatellite instability. J Nat Cancer Inst 2004; 96: 261-268.

22 Aretz S, Tricarico R, Papi L et al: MUTYH-associated polyposis (MAP): evidence for the origin of the common European mutations p.Tyr179Cys and p.Gly396Asp by founder events. Eur J Hum Genet 2014; 22: 923-929.

23 Nielsen M, de Miranda NF, van Puijenbroek M et al: Colorectal carcinomas in MUTYHassociated polyposis display histopathological similarities to microsatellite unstable carcinomas. BMC Cancer 2009; 9: 184. 
24 Vogt S, Jones N, Christian D et al: Expanded extracolonic tumor spectrum in MUTYHassociated polyposis. Gastroenterology 2009; 137: 1976-1985, e1-10.

25 Wasielewski M, Out AA, Vermeulen J et al: Increased MUTYH mutation frequency among Dutch families with breast cancer and colorectal cancer. Breast Cancer Res Treat 2010; 124: 635-641.

26 Chen JM: The 10-Mb paracentric inversion of chromosome arm $2 \mathrm{p}$ in activating MSH2 and causing hereditary nonpolyposis colorectal cancer: re-annotation and mutational mechanisms. Genes Chromosomes Cancer 2008; 47: 543-545.

27 Wagner A, van der Klift H, Franken $\mathrm{P}$ et al: A 10-Mb paracentric inversion of chromosome arm $2 \mathrm{p}$ inactivates $\mathrm{MSH} 2$ and is responsible for hereditary nonpolyposis colorectal cancer in a North-American kindred. Genes Chromosomes Cancer 2002; 35: 49-57.
28 Cooper DN, Chen JM, Ball EV et al: Genes, mutations, and human inherited disease at the dawn of the age of personalized genomics. Hum Mutat 2010; 31: 631-655.

29 Webb EL, Rudd MF, Houlston RS: Colorectal cancer risk in monoallelic carriers of MYH variants. Am J Hum Genet 2006; 79: 768-771, author reply 771-762.

30 Casper M, Plotz G, Juengling B, Trojan J, Lammert F, Raedle J: Adenoma development in a patient with MUTYH-associated polyposis (MAP): new insights into the natural course of polyp development. Dig Dis Sci 2010; 55: 1711-1715.

31 Strate LL, Syngal S: Hereditary colorectal cancer syndromes. Cancer Causes Control 2005; 16: 201-213.

32 Rodriguez-Soler M, Perez-Carbonell L, Guarinos C et al: Risk of cancer in cases of suspected lynch syndrome without germline mutation. Gastroenterology 2013; 144: 926-932, e921; quiz e913-924.

Supplementary Information accompanies this paper on European Journal of Human Genetics website (http://www.nature.com/ejhg) 